\title{
Black Phosphorus Radio-Frequency Transistors
}

\author{
Han Wang ${ }^{1, *}$, Xiaomu Wang ${ }^{2}$, Fengnian Xia ${ }^{2, *}$, Luhao Wang ${ }^{1}$, Hao Jiang ${ }^{3}$, \\ Qiangfei $\mathrm{Xia}^{3}$, Matthew L. Chin ${ }^{4}$, Madan Dubey ${ }^{4}$, Shu-jen $\mathrm{Han}^{5}$ \\ ${ }^{1}$ Ming Hsieh Department of Electrical Engineering, University of Southern California, \\ Los Angeles, CA 90089 \\ ${ }^{2}$ Department of Electrical Engineering, Yale University, New Haven, CT 06511 \\ ${ }^{3}$ Department of Electrical \& Computer Engineering, University of Massachusetts, \\ Amherst, MA 01003 \\ ${ }^{4}$ Sensors and Electron Devices Directorate, US Army Research Laboratory, \\ Adelphi, MD 20723 \\ ${ }^{5}$ IBM T. J. Watson Research Center, Yorktown Heights, NY 10598
}

\begin{abstract}
Few-layer and thin film forms of layered black phosphorus (BP) have recently emerged as a promising material for applications in high performance nanoelectronics and infrared optoelectronics. Layered BP thin film offers a moderate bandgap of around $0.3 \mathrm{eV}$ and high carrier mobility, leading to transistors with decent on-off ratio and high on-state current density. Here, we demonstrate the gigahertz frequency operation of black phosphorus field-effect transistors for the first time. The BP transistors demonstrated here show excellent current saturation with an on-off ratio exceeding $2 \times 10^{3}$. We achieved a current density in excess of $270 \mathrm{~mA} / \mathrm{mm}$ and DC transconductance above $180 \mathrm{mS} / \mathrm{mm}$ for hole conduction. Using standard high frequency characterization techniques, we measured a short-circuit current-gain cut-off frequency $f_{\mathrm{T}}$ of $12 \mathrm{GHz}$ and a maximum oscillation frequency $f_{\max }$ of $20 \mathrm{GHz}$ in $300 \mathrm{~nm}$ channel length devices. BP devices may offer advantages over graphene transistors for high frequency electronics in terms of voltage and power gain due to the good current saturation properties arising from their finite bandgap, thus enabling the future ubiquitous transistor technology that can operate in the multi-GHz frequency range and beyond.
\end{abstract}

*Email: han.wang.4@usc.edu, fengnian.xia@yale.edu 


\section{Introduction}

Typical channel materials in thin film electronics include amorphous or polycrystalline silicon ${ }^{1-3}$, organic compounds ${ }^{4,5}$, and oxides ${ }^{6,7}$. These materials usually exhibit a sizable bandgap but compromised carrier mobility, making them undesirable for radio-frequency $(\mathrm{RF})$ electronics. Early interests in using two-dimensional materials for RF thin film electronics focused mainly on graphene. Since the first demonstration of high frequency current gain in graphene field-effect transistors (FETs) in $2007^{8}$, graphene has inspired great interests for RF applications due to its high mobility, high carrier velocity and long mean free path ${ }^{9-14}$. However, several years of intensive research has revealed that graphene transistors might suffer from a few fundamental limitations that will restrict its high frequency performance. The key shortcoming of graphene RF transistors is their lack of current saturation as a result of graphene's zero-bandgap nature, that can lead to reduced voltage and power gains ${ }^{15,16}$, and to a lesser extent, the current gain ${ }^{17}$. As a result, most graphene RF FETs may have relatively high short-circuit current-gain cut-off frequency $\left(f_{\mathrm{T}}\right)$, but significantly lower maximum oscillation frequency $\left(f_{\max }\right)$, which benchmarks the power gain of the transistor. On the other hand, transistors based on transition metal dichalcogenides, such as molybdenum disulfide $\left(\mathrm{MoS}_{2}\right)$ and tungsten diselenide ( $\left.\mathrm{WSe}_{2}\right)$, only showed limited potential for high frequency applications because of their relatively low mobility ${ }^{18,19}$. Recently, layered black phosphorus (BP) thin film has emerged as a promising candidate for high performance thin film electronics due to its moderate bandgap of $0.3 \mathrm{eV}$ and high carrier mobility. The recent experiment has shown hole mobility exceeding $650 \mathrm{~cm}^{2} \mathrm{~V}^{-1} \mathrm{~s}^{-1}$ at room temperature and above $1,000 \mathrm{~cm}^{2} \mathrm{~V}^{-1} \mathrm{~s}^{-1}$ at $120 \mathrm{~K}$ along the light effective mass ( $\mathrm{x}$ ) 
direction ${ }^{20}$. While in bulk black phosphorus, mobilities exceeding $1,000 \mathrm{~cm}^{2} \mathrm{~V}^{-1} \mathrm{~s}^{-1}$ at room temperature for both electrons and holes, and exceeding $50,000 \mathrm{~cm}^{2} \mathrm{~V}^{-1} \mathrm{~s}^{-1}$ at $30 \mathrm{~K}$ have been demonstrated ${ }^{21}$. In this work, we demonstrate for the first time the operation of black phosphorus FETs in the gigahertz frequency range. Future ubiquitous transistor technologies using this novel layered material with high mobility and highly desirable current saturation property may revolutionize the electronic systems for many civilian and defense applications.

\section{Results}

\section{Device fabrication}

Orthorhombic bulk black phosphorus is an elemental layered material with $\mathrm{D}_{2 \mathrm{~h}}$ point group symmetry as shown in Fig. 1a. It is the most stable allotrope of phosphorus and the electronic properties of bulk BP have been studied several decades ago ${ }^{22-26}$. Recently, BP in its few-layer and thin-film form has inspired renewed interests among physicists and engineers due to its promising potential for application in thin film electronics and infrared optoelectronics ${ }^{20,27-29}$. The layered nature of BP crystals allows single- and multi-layer atomic crystals to be obtained through mechanical exfoliation techniques. In our experiments, multi-layer BP was first exfoliated using the standard micromechanical cleavage method from a bulk BP crystal, then transferred onto $300 \mathrm{~nm}$ thick silicon dioxide thermally grown on highly resistive silicon $\left(>10^{4} \Omega \cdot \mathrm{cm}\right)$. Atomic force microscope (AFM) can be used to determine the number of atomic layers in a BP flake, which has a layer-to-layer spacing of $0.53 \mathrm{~nm}^{20,21}$. The $\mathrm{x}$-, $\mathrm{y}$ - and z-directions of the crystal lattice are indicated in Fig. 1a. In a series of recent publications, the mobility 
and the on-off current ratio of BP FETs have been studied with respect to the thickness and layer number in the BP channel ${ }^{20,27,28}$. In this work, BP thin films with thicknesses around 6-10 $\mathrm{nm}$ were selected for the best balanced mobility and on-off properties for RF applications. Transistors using thinner BP films exhibit an on-off current ratio as high as $2 \times 10^{5}$ but low mobility of below $100 \mathrm{~cm}^{2} \mathrm{~V}^{-1} \mathrm{~s}^{-1}$ due to the scattering from the external environment. Utilization of excessively thick BP films can lead to higher mobility but a reduced on-off ratio and less pronounced current saturation. The inset of Fig. 1b shows the optical micrograph of a typical BP flake used for device fabrication. AFM measurements indicate a thickness of $8.5 \mathrm{~nm}$ in this flake (Fig. 1b). To enhance the channel mobility, crystal orientations of the flakes were first identified using either Raman spectroscopy or infrared spectroscopy techniques and the transistors were built along the light effective mass (x-) direction of the BP lattice. Fig. 1c shows the Raman spectrum of the BP flake with the excitation laser polarized along the $\mathrm{x}$-direction. The characteristic peaks at 470,440 , and $365 \mathrm{~cm}^{-1}$ correspond to $\mathrm{A}_{\mathrm{g}}^{2}, \mathrm{~B}_{2 \mathrm{~g}}$, and $\mathrm{A}_{\mathrm{g}}^{1}$ modes, respectively ${ }^{20,30}$. The $A_{g}^{2}$ mode has higher intensity compared to $B_{2 g}$, and $A_{g}^{1}$ modes with this particular excitation laser polarization. Fig. 1d shows the polarization-resolved infrared spectra of the flake and the x-direction can be clearly identified as the direction with the highest optical conductivity around the band edge $e^{20,21}$. The source and drain electrodes were formed with $1 \mathrm{~nm} \mathrm{Ti} / 20 \mathrm{~nm} \mathrm{Pd} / 30 \mathrm{~nm}$ Au metal stack, which favors ptype carrier injection into the channel owing to the large work-function $(\sim 5.22 \mathrm{eV})$ of Pd. The gate dielectric was made with $21 \mathrm{~nm}$ of $\mathrm{HfO}_{2}$ deposited by atomic layer deposition (ALD) technique at a temperature of $150{ }^{\circ} \mathrm{C}$. The typical dielectric constant is around 13 , determined by ellipsometric measurement on a control sample. Finally, the gate electrode 
was defined by electron-beam lithography to form transistors with sub-micrometer channel lengths. The fabrication process is described in more detail in Supporting Information. Fig. 2b shows the optical micrograph of the full layout of the device. The standard ground-signal-ground (GSG) pads were fabricated to realize signal transition from microwave coax cables to on-chip coplanar waveguide electrodes.

\section{Characterization}

Fig. 2a shows the schematic of the transistor structure. Fig. $2 \mathrm{c}$ and $2 \mathrm{~d}$ display the DC characteristics of a top-gated BP FET with a $300 \mathrm{~nm}$ channel length $\left(\mathrm{L}_{\mathrm{G}}\right)$. The device was built along the $\mathrm{x}$-direction of the $8.5 \mathrm{~nm}$ thick flake shown in Fig. 1. Fig. 2c shows the measured drain current $\left(I_{\mathrm{DS}}\right)$ as a function of drain-source bias voltage $\left(V_{\mathrm{DS}}\right)$ at gate bias $\left(V_{\mathrm{GS}}\right)$ from $0 \mathrm{~V}$ to $-2 \mathrm{~V}$ in steps of $-0.5 \mathrm{~V}$. The current saturation in the $\mathrm{BP}$ transistor is clearly visible and significantly improved from that in most graphene FETs due to the finite bandgap in BP thin film. This is typical for a BP device with $\sim 8.5 \mathrm{~nm}$ channel thickness and agrees well with previous demonstrations ${ }^{20}$. Good current saturation characteristics will lead to low output conductance defined as $g_{0}=\left.\frac{\mathrm{d} I_{\mathrm{DS}}}{\mathrm{d} V_{\mathrm{DS}}}\right|_{V_{\mathrm{GS}}=\text { constant }}$, which is the differential drain current change with respect to the variation in drain voltage bias. Low output conductance is critical for improving voltage and power gains, and to a lesser extent, the current gain in RF transistors. As reported in our previous work ${ }^{20}$, typical Hall mobility along X-direction of BP flakes with thickness around $8 \mathrm{~nm}$ is above $400 \mathrm{~cm}^{2} \mathrm{~V}^{-}$ ${ }^{1} \mathrm{~s}^{-1}$ at room temperature. Fig. $2 \mathrm{~d}$ shows $I_{\mathrm{DS}}$ as a function of $V_{\mathrm{GS}}$ at $V_{\mathrm{DS}}=-2 \mathrm{~V}$, where on-

off current ratio over $2 \times 10^{3}$ is achieved. The as-fabricated device shows p-type conduction with threshold voltage around -0.7 V. A key factor influencing the high- 
frequency small signal response of a transistor is its transconductance $\left(g_{\mathrm{m}}\right)$, defined as the first derivative of the transfer characteristics, i.e. $g_{\mathrm{m}}=\left.\frac{\mathrm{d} I_{\mathrm{DS}}}{\mathrm{d} V_{\mathrm{GS}}}\right|_{V_{\mathrm{DS}}=\text { constant }}$. The inset of Fig. $2 \mathrm{~d}$ shows the measured $g_{\mathrm{m}}$ as a function of the gate voltage at $V_{\mathrm{DS}}=-2 \mathrm{~V}$. The peak value of this extrinsic $g_{\mathrm{m}}$ exceeds $180 \mathrm{mS} / \mathrm{mm}$ at $V_{\mathrm{G}}=-1.75 \mathrm{~V}$ while the peak on-state current density measured exceeds $270 \mathrm{~mA} / \mathrm{mm}$ at $V_{\mathrm{DS}}=-2 \mathrm{~V}$ and $V_{\mathrm{GS}}=-2.5 \mathrm{~V}$. Fig. $2 \mathrm{~d}$ also shows $I_{\mathrm{DS}}$ as a function of top-gate voltage $V_{\mathrm{GS}}$ at a drain bias of $V_{\mathrm{DS}}=-2 \mathrm{~V}$ with the current plotted in logarithmic scale. The on-off current ratio of the device exceeds $2 \times 10^{3}$. Hence, the BP transistor demonstrated here shows significant advantages over graphene transistors in terms of its current saturation properties and on-off current ratio. Typical monolayer graphene devices have on-off current ratio less than 10 at room temperature. In bilayer graphene devices, the on-off current ratio only reaches around 100 even with bandgap opening induced by a strong external electrical field ${ }^{31}$.

\section{RF Characterization}

To characterize the high frequency performance of BP RF transistors, we used the standard S-parameter measurement with on-chip probing utilizing GSG probes and Agilent N5230 vector network analyzer up to $50 \mathrm{GHz}$. Key figures of merit for microwave transistors can then be obtained for the BP devices. The network analyzer and the entire testing fixture were first calibrated using standard open, short, and load calibrations. Standard open and short structures were then used to de-embed the signals of the parallel and series parasitics associated with the measurement pads and connections $^{15,32-34}$. The measurement procedure used in this work followed strictly the standard calibration and de-embedding processes widely accepted in the semiconductor 
industry, where the calibration step moves the reference plane to the tips of the GSG probes and the de-embedding step gives access to the performance of the active device region.

Fig. 3a and $3 b$ plot the short-circuit current gain $\left(h_{21}\right)$, the unilateral power gain (U) and maximum stable gain (MSG)/maximum available gain (MAG) extracted from Sparameters before and after de-embedding, respectively, measured at $V_{\mathrm{DS}}=-2.0 \mathrm{~V}$ and $V_{\mathrm{GS}}=-1.7 \mathrm{~V}$ for the device with $\mathrm{L}_{\mathrm{G}}=300 \mathrm{~nm}$. The plot of $\left|\mathrm{h}_{21}\right|^{2}$ follows the characteristic $1 / f$ relation with respect to frequency at a $20 \mathrm{~dB} / \mathrm{dec}$ slope $^{16}$. Fig. $3 \mathrm{a}$ and $3 \mathrm{~b}$ show that the $300 \mathrm{~nm}$ channel length device has a $f_{\mathrm{T}}$ of $7.8 \mathrm{GHz}$ before de-embedding and $12 \mathrm{GHz}$ after de-embedding, as extracted from the frequencies at which $\left|\mathrm{h}_{21}\right|$ reaches unity. Gummel's method provides another way of extracting the cut-off frequency ${ }^{35}$ where $f_{\mathrm{T}}$ is extracted from the reciprocal of the initial slope in the imaginary part of $1 / h_{21}$ vs. frequency plot. The values of $f_{\mathrm{T}}$ obtained by both $1 / f$ extraction and Gummel's method match closely for both $300 \mathrm{~nm}$ (Fig. 3c and 3d) and $1 \mu \mathrm{m}$ (see Fig. S1 in the Supporting Information) channel length devices before and after de-embedding.

While $f_{\mathrm{T}}$ is an important figure of merit related to the intrinsic speed of the $\mathrm{BP}$ transistor, another key figure of merit for analog application is the maximum oscillation frequency. It is the highest possible operating frequency at which a transistor can still amplify power. $f_{\max }$ can be extracted from $\mathrm{U}$ or MSG/MAG of the device ${ }^{16,36}$. The unilateral power gain, U, also known more generally as Mason's U invariant, is a key parameter for any general two-port network. It carries great significance as an invariant parameter of the system under linear, lossless and reciprocal transformations. In Mason's classic work ${ }^{37,38}$, the rich physical meaning of $U$ was interpreted in three different ways 
as a maximum power gain, as a device activity measure, and as an invariant under a class of bilinear Möbius transformations. In transistor characterizations, $U$ is the power gain under the condition of (1) Unilateralization, and (2) Conjugate matched load for maximum power transfer. In Fig. $3 \mathrm{a}$ and $3 \mathrm{~b}$, the plots of $\mathrm{U}$ follow a $20 \mathrm{~dB} / \mathrm{dec}$ slope, and both $\mathrm{U}$ and MSG/MAG plots give similar $f_{\max }$ of $12 \mathrm{GHz}$ before de-embedding and 20 $\mathrm{GHz}$ after de-embedding, respectively.

The $300 \mathrm{~nm}$ channel length device has an extrinsic $f_{\mathrm{T}} \cdot \mathrm{L}_{\mathrm{G}}$ product of $3.6 \mathrm{GHz} \mu \mathrm{m}$. Using a slightly different design of the open pattern where the gate electrode in the "open" structure extends into the spacing between the source and drain electrodes ${ }^{15}$, we can eliminate most of the gate-source and gate-drain parasitic capacitances $C_{\mathrm{gs}}$ and $C_{\mathrm{gd}}$. This will allow the extraction of a new $f_{\mathrm{T}}$ value that reflects the more intrinsic property of the BP channel, and an intrinsic $f_{\mathrm{T}}$ value $\left(f_{\mathrm{T}, \mathrm{int}}\right)$ close to $51 \mathrm{GHz}$ is obtained for the same device, which corresponds to the average saturation velocity in the channel approximately equal to $v_{\mathrm{sat}}=2 \pi f_{\mathrm{T}} \cdot L_{\mathrm{G}} \sim 9.6 \times 10^{6} \mathrm{~cm} / \mathrm{s}$. However, we would like to emphasize that $f_{\mathrm{T} \text {,int }}$ only represents the upper limit of the possible frequency spectrum for this transistor. In any practical applications, the $C_{\mathrm{gs}}$ and $C_{\mathrm{gd}}$ of the device always significantly affect the device performance. As a result, we report $f_{\mathrm{T}}=12 \mathrm{GHz}$ and $f_{\max }=20$ $\mathrm{GHz}$ in Fig. 3 as the practically operable cut-off frequencies of the active device region $^{15,33}$, which are extracted based on standard characterization techniques commonly used for the characterization in silicon and III-V high frequency transistors. The intrinsic cut-off frequency of $51 \mathrm{GHz}$ is extracted only as a way to approximately estimate the saturation velocity and it may not be appropriate for technology benchmarking. The RF characteristics for a device with $1 \mu \mathrm{m}$ channel length are also reported in the Supporting 
Information. The $1 \mu \mathrm{m}$ channel length device has peak $f_{\mathrm{T}}=2.8 \mathrm{GHz}$ and $f_{\max }=5.1 \mathrm{GHz}$ before de-embedding and $f_{\mathrm{T}}=3.3 \mathrm{GHz}$ and $f_{\max }=5.6 \mathrm{GHz}$ after de-embedding (see Fig. $\mathrm{S} 1$ in the Supporting Information).

The intrinsic limit of the cut-off frequency can be estimated using $f_{T}=\frac{g_{m}}{2 \pi C_{g s}}$. Assuming the same $g_{m}$ and gate dielectrics property from the measured data, an $f_{\mathrm{T}}$ above $100 \mathrm{GHz}$ may be reached if the channel length reduces to $30 \mathrm{~nm}$. On the other hand, $f_{\max }$ depends on both the current and voltage gains. It is related to $f_{\mathrm{T}}$, following $f_{\max }=\frac{f_{T}}{2} \sqrt{\frac{r_{0}}{R_{g}+R_{i}}}$. We can see that a high $f_{\mathrm{T}}$ will enhance $f_{\max }$ and good current saturation, i.e. high output resistance $\left(r_{0}\right)$, low gate resistance $\left(R_{\mathrm{g}}\right)$ and low input resistance $\left(R_{\mathrm{i}}\right)$ are critical for improving $f_{\max }$ of the device.

Fig. 4 shows the magnitude of the small-signal open-circuit voltage gain $\left|z_{21} / z_{11}\right|$ as a function of frequency before and after de-embedding. An open-circuit voltage gain refers to the voltage gain subject to infinitely high load impedance. Here, the voltage gain is obtained from the ratio between the open circuit forward transfer impedance $z_{21}$ and the open circuit input impedance $\mathrm{z}_{11}$. Since $z_{11}=\left.\frac{v_{1}}{i_{1}}\right|_{i_{2}=0}$ and $z_{21}=\left.\frac{v_{2}}{i_{1}}\right|_{i_{2}=0}$, the ratio $z_{21} / z_{11}$ is equal to $\left.\frac{v_{2}}{v_{1}}\right|_{i_{2}=0}$, i.e. the voltage gain with the output port in open condition. $v_{1}$ and $v_{2}$ refer to the voltages at the input and output ports, respectively. In transistors, $v_{1}$ is the small-signal input voltage at the gate and the $v_{2}$ is the small-signal output voltage at the drain. Based on the definition of $g_{\mathrm{m}}$ and $g_{0}$ discussed earlier, we can see that the voltage gain $\left|z_{21} / z_{11}\right|$ is closely related to the ratio $g_{m} / g_{0}$. Devices with good current saturation and high transcondutance are hence expected to have high voltage gains. The BP transistors show good voltage gain characteristics. As shown in Fig. 4, the before-de-embedding 
voltage gain stays above unity $(0 \mathrm{~dB})$ up to $13 \mathrm{GHz}$. The after-de-embedding voltage gain is close to $20 \mathrm{~dB}$ at $2 \mathrm{GHz}$ and stays above unity $(0 \mathrm{~dB})$ for the entire frequency range measured up to $50 \mathrm{GHz}$. For a longer channel length $(1 \mu \mathrm{m})$ device (see Fig. S2 in the Supporting Information), the before-de-embedding voltage gain is above unity ( $0 \mathrm{~dB})$ up to $10 \mathrm{GHz}$. The after-de-embedding voltage gain is above $15 \mathrm{~dB}$ at $1 \mathrm{GHz}$ and stays above unity $(0 \mathrm{~dB})$ up to $30 \mathrm{GHz}$.

\section{Summary}

In this work, we investigated the high frequency characteristics of black phosphorus field effect transistors, whose channels were fabricated along the light effective mass ( $\mathrm{x}-$ ) direction. The device operates well into the $\mathrm{GHz}$ frequency range of the radio frequency spectrum. We carried out standard S-parameter measurements to characterize the high frequency response of these top-gated BP transistors. The shortcircuit current gain, maximum stable gain/maximum available gain, unilateral power gain and voltage gain of the devices were carefully extracted. The short-circuit current gain of BP transistors shows the $20 \mathrm{~dB} / \mathrm{dec} 1 / f$ frequency dependence at high frequency. We measured a peak short-circuit current gain cutoff frequency $f_{\mathrm{T}}$ of $12 \mathrm{GHz}$ and maximum oscillation frequency $f_{\max }$ of $20 \mathrm{GHz}$ for a $300 \mathrm{~nm}$ channel length $\mathrm{BP}$ transistor, demonstrating the $\mathrm{GHz}$ operation of $\mathrm{BP}$ devices for the first time. These results clearly reveal the potentials of $\mathrm{BP}$ transistors to function as power and voltage amplifiers in multi-GHz frequency analogue and digital electronics demanded by many emerging civilian and military applications. 


\section{References}

1. Powell, M. J. The physics of amorphous-silicon thin-film transistors. IEEE Trans. Electron Devices, 36, 2753-2763 (1989).

2. Street, R. A. Thin-Film Transistors. Adv. Mater., 21, 2007-2022 (2009).

3. Hawkins, W. G. Polycrystalline-silicon device technology for large-area electronics. IEEE Trans. Electron Devices, 33, 477-481 (1986).

4. Forrest, S. R. The path to ubiquitous and low-cost organic electronic appliances on plastic. Nature, 428, 911-918 (2004).

5. Dimitrakopoulos, C. D., Mascaro, D. J. Organic thin-film transistors: A review of recent advances. IBM J. Res. Dev., 45, 11-27 (2004).

6. Nomura, K. et al. Amorphous Oxide Semiconductors for High-Performance Flexible Thin-Film Transistors. Japanese Journal of Applied Physics, 45, 4303 (2006).

7. Carcia, P. F., McLean, R. S., Reilly, M. H. \& Nunes, G. Jr., Transparent ZnO thin-film transistor fabricated by RF magnetron sputtering. Appl. Phys. Lett., 82, 1117 (2003);

8. Meric, I., Baklitskaya, N., Kim, P. \& Shepard, K. L. RF performance of top-gated, zero-bandgap graphene field-effect transistors. IEEE IEDM Tech. Digest, 2008.

9. Lin, Y.-M., Dimitrakopoulos, C. D., Jenkins, K. A., Farmer, D. B., Chiu, H.-Y., Grill, A. \& Avouris, P. 100-GHz Transistors from Wafer-Scale Epitaxial Graphene. Science, 327, 662 (2010).

10. Wu, Y., Lin, Y., Bol, A. A., Jenkins, K. A., Xia, F., Farmer, D. B., Zhu, Y., \& Avouris, P. High-frequency, scaled graphene transistors on diamond-like carbon. Nature, 472, 74-78 (2011).

11. Liao, L. et al. High-speed graphene transistors with a self-aligned nanowire gate. Nature, 467, 305-308 (2010).

12. Wang, H., Nezich, D., Kong, J. \& Palacios, T. Graphene frequency multipliers. IEEE Elec. Dev. Lett., 30, 547-549 (2009).

13. Wang, H., Hsu, A., Wu, J., Kong, J. \& Palacios, T. Graphene-based ambipolar RF mixers. IEEE Elec. Dev. Lett., 31, 906-908 (2010). 
14. Han, S.-J., Valdes-Garcia, A., Oida, S., Jenkins, K. A. \& Haensch, W. Graphene radio frequency receiver integrated circuit. Nat. Commun., 5:3086 doi:10.1038/ncomms4086 (2014).

15. Wang, H. Chapter 4. In Two-Dimensional Materials for Electronic Applications. Ph.

D. Thesis, Massachusetts Institute of Technology, Cambridge, MA, USA, 2014.

http://dspace.mit.edu/handle/1721.1/84899

16. Schwierz, F. \& Liou, J. J. Modern Microwave Transistors: Theory, Design, and

Performance, Wiley-Interscience, 1st edition, 2002.

17. Tasker, P. J. \& Hughes, B. Importance of source and drain resistance to the maximum $f_{\mathrm{T}}$ of millimeter-wave MODFETs. IEEE Elec. Dev. Lett., 10, 291-293 (1989).

18. Zhu, W., Low, T., Lee, Y.-H., Wang, H., Farmer, D. B., Kong, J., Xia, F. \& Avouris,

P. Electronic transport and device prospects of monolayer molybdenum disulphide grown by chemical vapour deposition. Nat. Commun., 5:3087 doi:10.1038/ncomms4087 (2014).

19. Huang, J.-K. et al. Large-Area Synthesis of Highly Crystalline $\mathrm{WSe}_{2}$ Monolayers and Device Applications, ACS Nano, 8, 923-930 (2014).

20. Xia, F., Wang, H. \& Jia, Y. Rediscovering black phosphorus as an anisotropic layered material for optoelectronics and electronics. Nat. Commun., 5:4458

doi:10.1038/ncomms5458 (2014).

21. Morita, A. Semiconducting black phosphorus. Appl. Phys. A, 39, 227-242, 1986.

22. Keyes, R. The electrical properties of black phosphorus. Phys. Rev. 92, 580-584 (1953).

23. Warschauer, D. Electrical and optical properties of crystalline black phosphorus. $J$. Appl. Phys. 34, 1853-1860 (1963).

24. Jamieson, J. Crystal structures adopted by black phosphorus at high pressures. Science 139, 1291-1292 (1963).

25. Wittig, J. \& Matthias, B. T. Superconducting phosphorus. Science 160, 994-995 (1968).

26. Maruyama, Y., Suzuki, S., Kobayashi, K. \& Tanuma, S. Synthesis and some properties of black phosphorus single crystals. Physica, 105B, 99-102 (1981). 
27. Li, L. et al. Black phosphorus field-effect transistors. Nat. Nanotechnol., 9, 372-377 (2014).

28. Liu, H. et al. Phosphorene: an unexplored 2D semiconductor with a high hole mobility. ACS Nano, 8, 4033-4041 (2014).

29. Koenig, S., Doganov, R., Schmidt, H., Castro Neto, A. \& Ozyilmaz, B. Electric field effect in ultrathin black phosphorus. Appl. Phys. Lett., 104, 103106 (2014).

30. Akahama, Y., Kobayashi, M. \& Kawamura, H. Raman study of black phosphorus up to $13 \mathrm{GPa}$. Solid State Comm. 104, 311-315 (1997).

31. Xia, F., Farmer, D. B., Lin, Y.-M., Avouris, P. Graphene field-effect transistors with high on/off current ratio and large transport band gap at room temperature. Nano Lett., 10, 715-718 (2010).

32. R. J. Collier and A. D. Skinner, Microwave Measurements, 3rd Edition, the Institution of Engineering and Technology, 2007.

33. Koolen, M. C. A., Geelen, J. A. \& Versleijen, M. P. J. An improved de-embedding technique for on-wafer high-frequency characterization. Proceedings of the Bipolar Circuits and Technology Meeting 1991, 188-191 (1991).

34. Kim, J.-Y., Choi, M.-K. \& Lee, S.-H. A ‘Thru-Short-Open’ De-embedding Method for Accurate On-Wafer RF Measurements of Nano-Scale MOSFETs. JSTS:Journal of Semiconductor Technology and Science, 12, 53-58 (2012).

35. Gummel, H. K. On the definition of the cutoff frequency $f_{\mathrm{T}}$. Proceedings of the IEEE, 57, 12, 2159-2159 (1969).

36. S-Parameter Techniques, HP Application Note 95-1, http://www.hparchive.com/Application_Notes/HP-AN-95-1.pdf.

37. Mason, S. J. Power gain in feedback amplifiers. Trans. IRE Professional Group on Circuit Theory, CT-1, $20-25$ (1954). (This work was previously reported in Tech. Rep. No. 257, Research Laboratory of Electronics, MIT, Cambridge, MA, 1953.

38. Gupta, M. S., Power Gain in Feedback Amplifiers, a Classic Revisited, IEEE Trans. Microwave Theory and Techniques, 40, 5 (1992). 


\section{Competing financial interests}

Authors declare no competing financial interests.

\section{Supporting Information}

Information on the device fabrication process and characterization method, additional high frequency characterization data are included in the Supporting Information. This material is available free of charge via the Internet at http://pubs.acs.org.

\section{Additional information}

Correspondence and requests for materials should be addressed to H.W.

(han.wang.4@usc.edu) and F.X. (fengnian.xia@yale.edu)

\section{Figure Captions}

\section{Figure 1. Characterization of the black phosphorus (BP) thin film.}

(a) Layered crystal structure of black phosphorus. The spacing between adjacent layers is

$5.3 \AA$ A. (b) atomic force microscope (AFM) data showing the thickness of a BP flake. The inset shows the optical micrograph of the BP flake with thickness around $8.5 \mathrm{~nm}$. (c) Raman spectrum of the BP flake along x-direction. (d) Polarization-resolved infrared spectra of a BP flake.

\section{Figure 2. DC characteristics of BP transistors.}

(a) Schematic of the BP transistor device structure. (b) Optical micrograph of the fabricated device. (c) Output characteristics of the BP transistor. $L_{\mathrm{G}}=300 \mathrm{~nm}$. (d) Transfer characteristics of the same BP transistor plotted in both linear and logarithmic 
scale. $V_{\mathrm{DS}}=-2 \mathrm{~V}$. The device has an on-off current ratio exceeding $2 \times 10^{3}$. The inset shows the transconductance $\mathrm{g}_{\mathrm{m}}$ of the device.

Figure 3. Current and power gain in BP transistors at GHz frequency.

(a) and (b) the short-circuit current gain $\mathrm{h}_{21}$, the maximum stable gain and maximum available gain MSG/MAG and the unilateral power gain $\mathrm{U}$ of the $300 \mathrm{~nm}$ channel length device before and after de-embedding, respectively. The device has $f_{\mathrm{T}}=7.8 \mathrm{GHz}, f_{\max }=12$ GHz before de-embedding, and $f_{\mathrm{T}}=12 \mathrm{GHz}, f_{\max }=20 \mathrm{GHz}$ after de-embedding. (c) and (d) the imaginary part of $1 / \mathrm{h}_{21}$ as a function of frequency before and after de-embedding, respectively. Based on Gummel's method, the initial slope of the curve is equal to $1 / f_{\mathrm{T}}$.

\section{Figure 4. Open-circuit voltage gain in BP transistors at GHz frequency}

The open-circuit voltage gain $\left(\mathrm{z}_{21} / \mathrm{z}_{11}\right)$ before and after de-embedding is shown as a function of the frequency. After de-embedding, the voltage gain stays close to $20 \mathrm{~dB}$ up to $2 \mathrm{GHz}$ and is above unity $(0 \mathrm{~dB})$ in the entire measurement range up to $50 \mathrm{GHz}$. The grey dashed line is a guide to the eyes. 
a

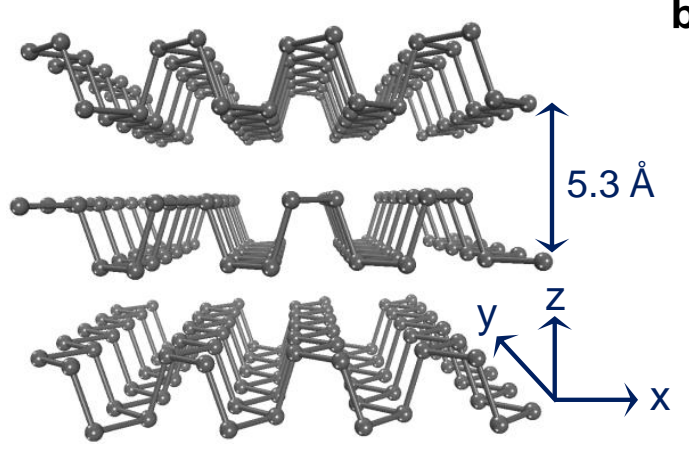

C

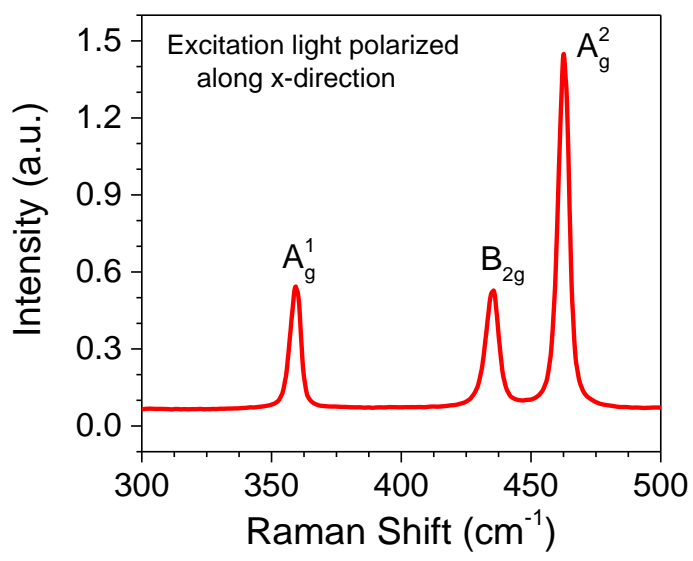

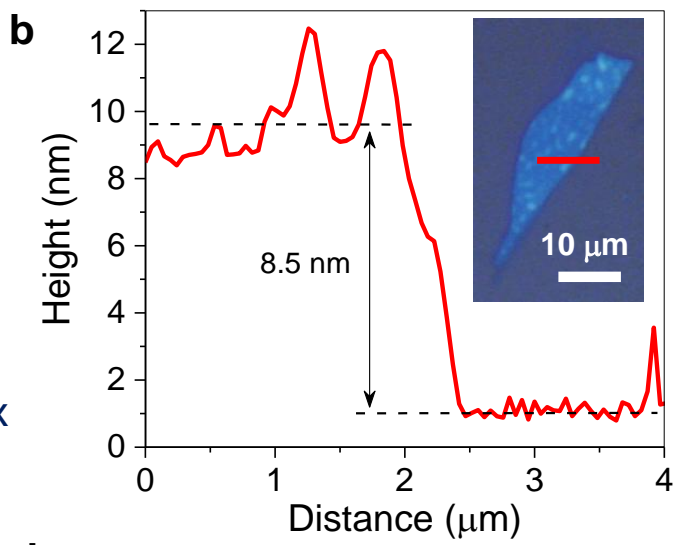

d

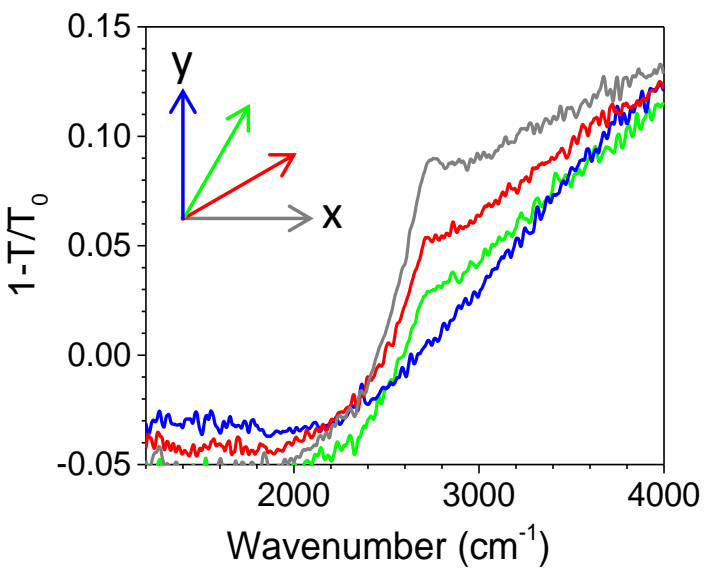


Figure 2. DC characteristics of BP transistors

a

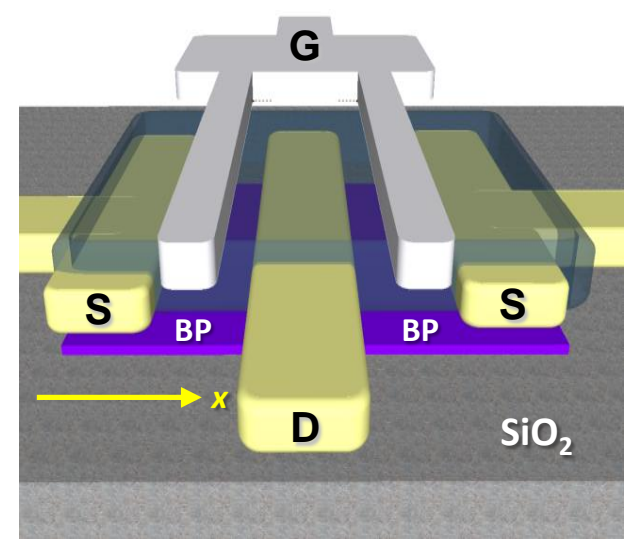

b

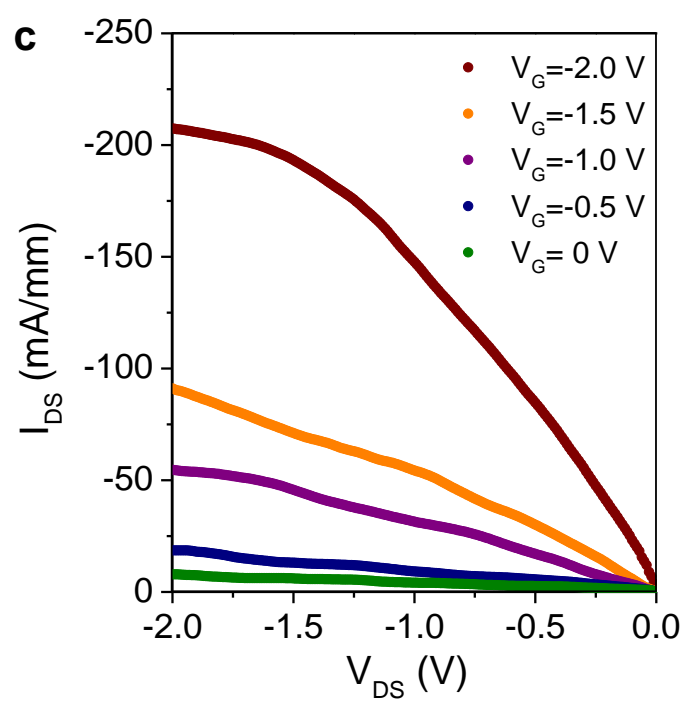

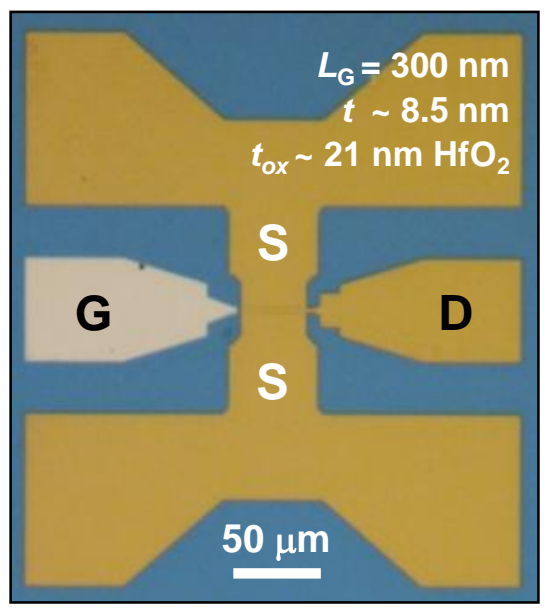

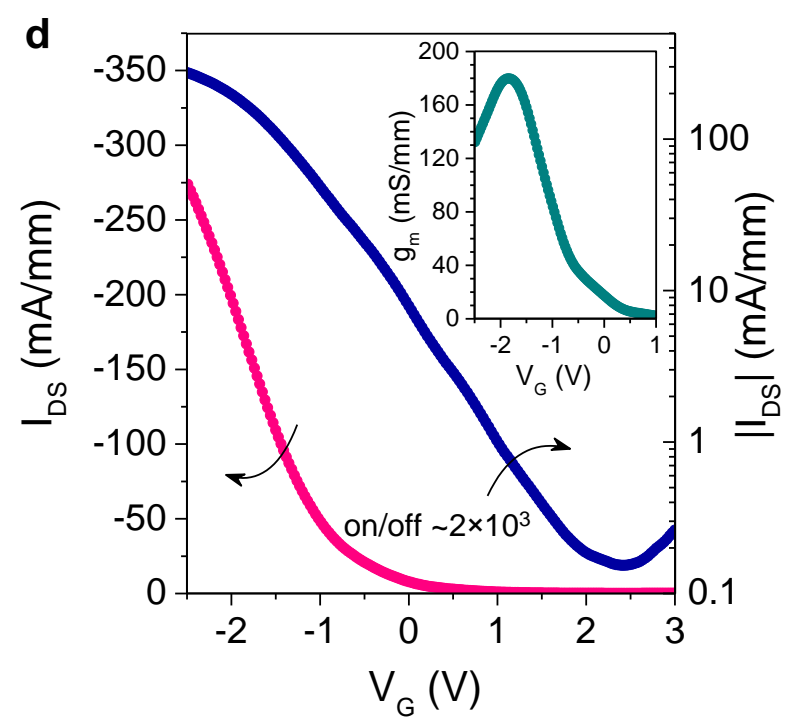


Figure 3. Current and power gain in BP transistors at GHz frequency
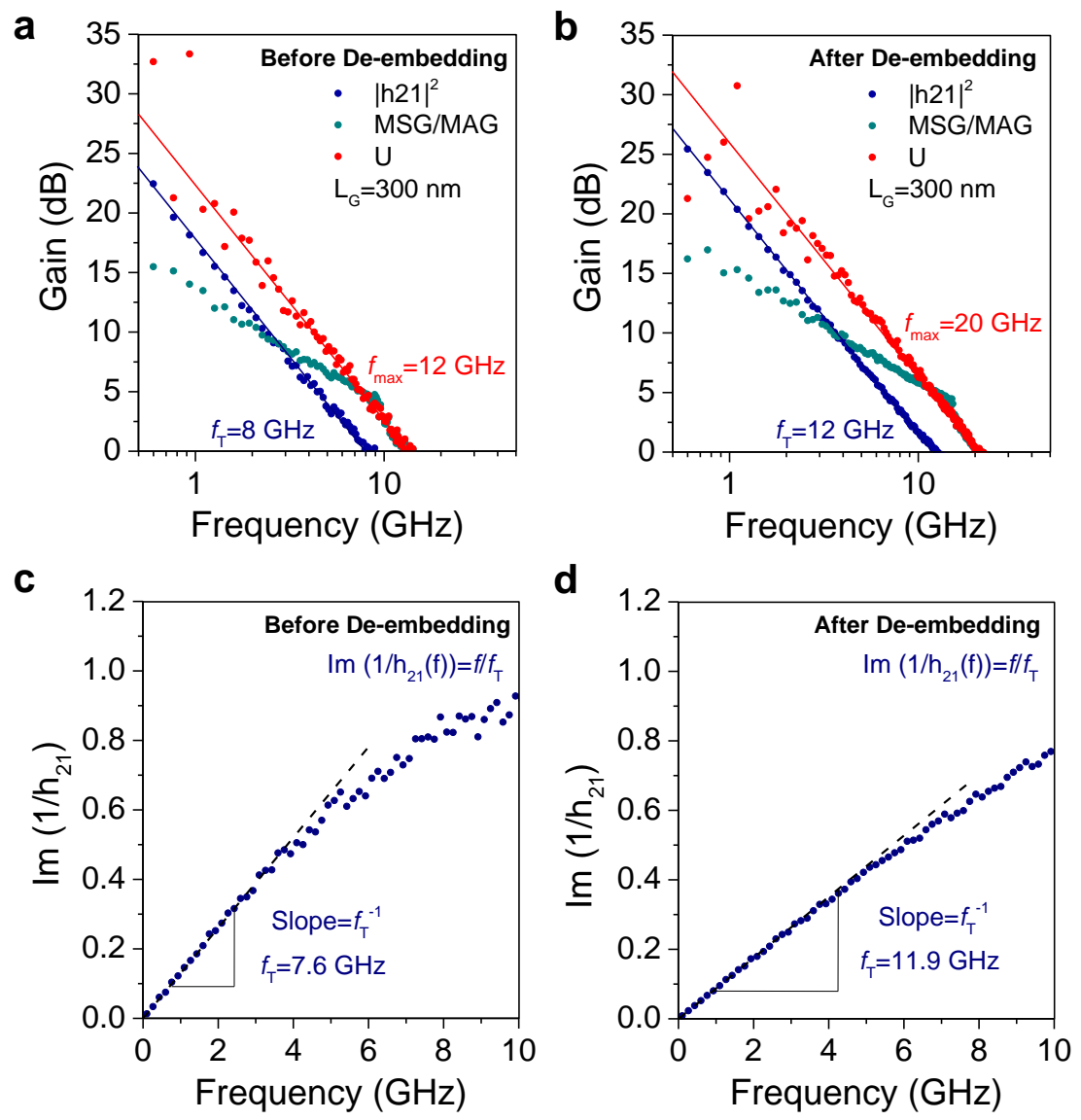
Figure 4. Open-circuit voltage gain in BP transistors at GHz frequency

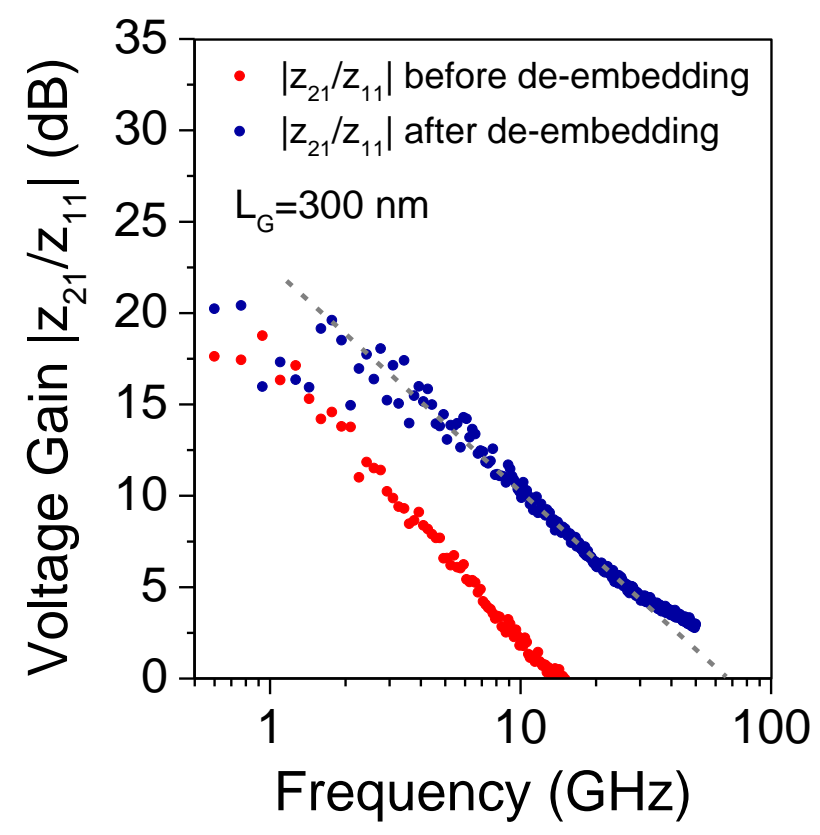




\title{
Supporting Information for
}

\section{Black Phosphorus Radio-Frequency Transistors}

\author{
Han Wang ${ }^{1, *}$, Xiaomu Wang ${ }^{2}$, Fengnian Xia ${ }^{2, *}$, Luhao Wang ${ }^{1}$, Hao Jiang ${ }^{3}$, \\ Qiangfei Xia ${ }^{3}$, Matthew L. Chin ${ }^{4}$, Madan Dubey ${ }^{4}$, Shu-jen Han $^{5}$ \\ ${ }^{1}$ Ming Hsieh Department of Electrical Engineering, University of Southern California, \\ Los Angeles, CA 90089 \\ ${ }^{2}$ Department of Electrical Engineering, Yale University, New Haven, CT 06511 \\ ${ }^{3}$ Department of Electrical \& Computer Engineering, University of Massachusetts, \\ Amherst, MA 01003 \\ ${ }^{4}$ Sensors and Electron Devices Directorate, US Army Research Laboratory, \\ Adelphi, MD 20723 \\ ${ }^{5}$ IBM T. J. Watson Research Center, Yorktown Heights, NY 10598
}

"Email: han.wang.4@usc.edu, fengnian.xia@yale.edu

\section{Methods}

Top-gated transistor fabrication. The fabrication of our devices starts with the exfoliation of BP thin films from bulk BP crystals onto $300 \mathrm{~nm} \mathrm{SiO} 2$ on a Si substrate, which has pre-patterned alignment grids, using the micro-mechanical cleavage technique. The thickness of the $\mathrm{SiO}_{2}$ was selected to provide the optimal optical contrast for locating BP flakes relative to the alignment grids. The thickness of BP layers was measured by atomic force microscopy (AFM). The next step was to pattern the resist layer for metallization using a Vistec $100 \mathrm{kV}$ electron-beam lithography system based on poly (methyl methacrylate) (950k MW PMMA). PMMA A3 was spun on wafer at a speed of $3000 \mathrm{rpm}$ for 1 minute and was then bakes at 175 degrees for 3 minutes. The dose for exposure is $1100 \mu \mathrm{C} \mathrm{cm}^{-2}$. Development was performed in 1:3 MIBK: IPA (Methyl isobutyl ketone: Isopropanol) for $90 \mathrm{~s}$. We then evaporated $1 \mathrm{~nm} \mathrm{Ti} / 20 \mathrm{~nm} \mathrm{Pd} / 30 \mathrm{~nm} \mathrm{Au}$ 
followed by lift-off in acetone to form the contacts. The $\mathrm{HfO}_{2}$ gate dielectric is deposited using atomic layer deposition (ALD) at $150{ }^{\circ} \mathrm{C}$. The gate electrode is also patterned using Vistec $100 \mathrm{kV}$ electron-beam lithography system.

AFM. Atomic force microscopy (AFM) for identifying the thin film thickness was performed on a Digital Instruments/Veeco Dimension 3000 system.

IR spectroscopy. Bruker Optics Fourier Transfer Infrared spectrometer (Vertex 70) integrated with a Hyperion 2000 microscope system was used to measure the infrared spectroscopy of the BP flakes in the $800 \mathrm{~cm}^{-1}$ to $4000 \mathrm{~cm}^{-1}$ range. The linear polarization of the incident light was achieved using an infrared polarizer.

Electrical characterization. DC electrical characterizations were performed using an Agilent B1500 semiconductor parameter analyzer and a Lakeshore cryogenic probe station with micromanipulation probes. The high frequency characterizations were performed using an Agilent N5230A Vector Network Analyzer.

\section{High frequency characterization of BP transistor with $1 \mu \mathrm{m}$ channel length}

The short-circuit current and power gain of a BP transistor with $1 \mu \mathrm{m}$ channel length are shown in Fig. $\mathrm{S} 1$. The open-circuit voltage gain $\mathrm{z}_{21} / \mathrm{z}_{11}$ both before and after de-embedding are shown in Fig. S2. The device has $f_{\mathrm{T}}=2.8 \mathrm{GHz}, f_{\mathrm{max}}=5.1 \mathrm{GHz}$ before de-embedding, and $f_{\mathrm{T}}=3.3 \mathrm{GHz}, f_{\max }=5.6 \mathrm{GHz}$ after de-embedding. After de-embedding, the voltage gain stays above $15 \mathrm{~dB}$ up around $1 \mathrm{GHz}$ and is above unity $(0 \mathrm{~dB})$ up to $30 \mathrm{GHz}$. 

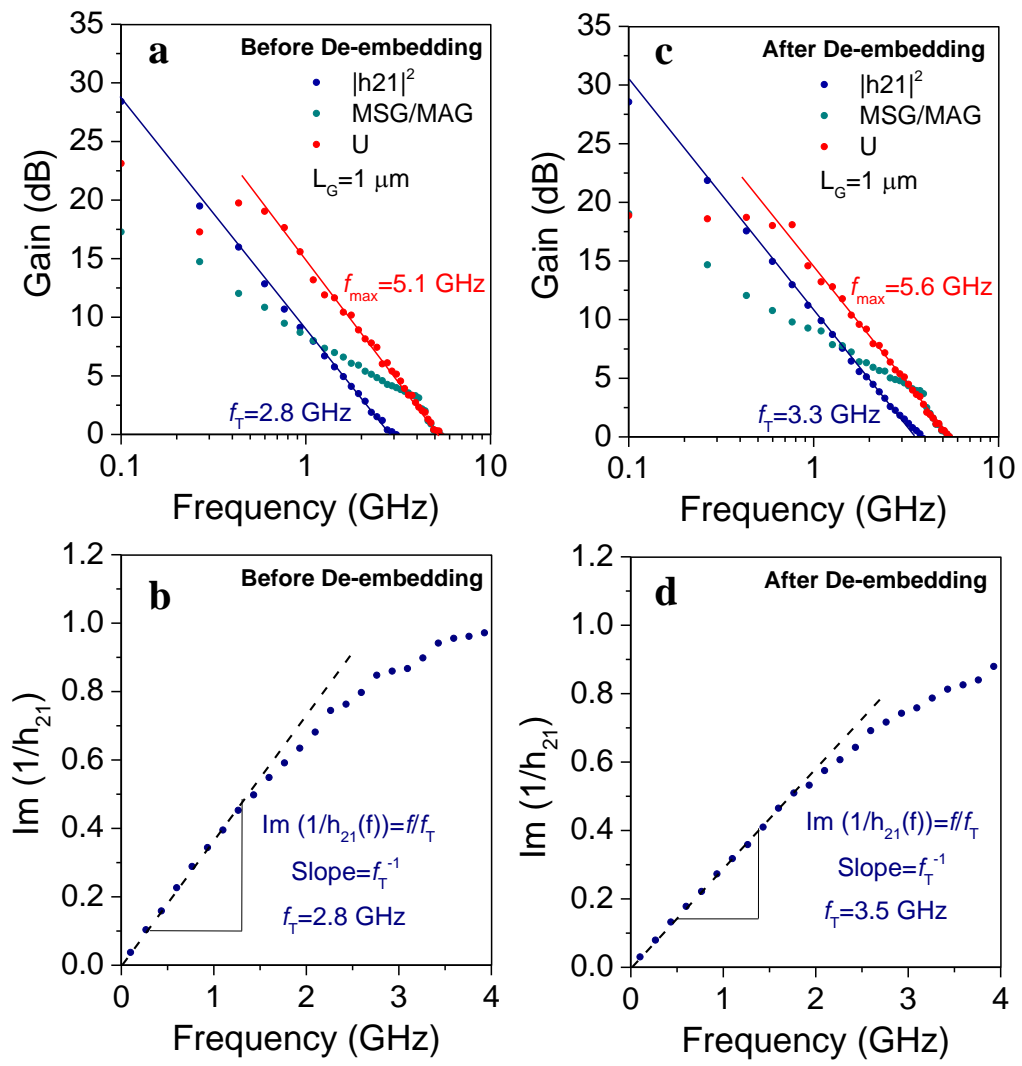

Fig. S1 (a) and (b) the short-circuit current gain $\mathrm{h}_{21}$, the maximum stable gain and maximum available gain MSG/MAG and the unilateral power gain $U$ of the device with $1 \mu \mathrm{m}$ channel length before and after deembedding, respectively. The device has $f_{\mathrm{T}}=2.8 \mathrm{GHz}, f_{\max }=5.1 \mathrm{GHz}$ before de-embedding, and $f_{\mathrm{T}}=3.3 \mathrm{GHz}$, $f_{\max }=5.6 \mathrm{GHz}$ after de-embedding. (c) and (d) the imaginary part of $1 / \mathrm{h}_{21}$ as a function of frequency before and after de-embedding, respectively. Based on Gummel's method, the initial slope of the curve is equal to $1 / f_{\mathrm{T}}$.

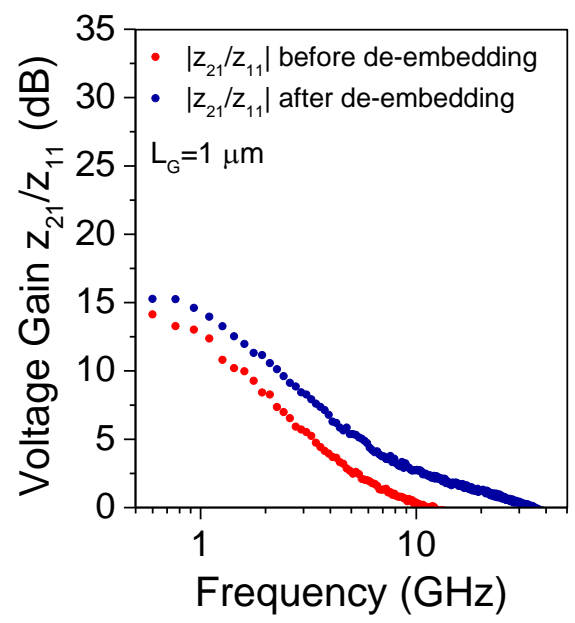

Fig. S2 the open-circuit voltage gain $\left(\mathrm{z}_{21} / \mathrm{z}_{11}\right)$ before and after de-embedding is shown as a function of the frequency. After de-embedding, the voltage gain stays above $15 \mathrm{~dB}$ up to around $1 \mathrm{GHz}$ and is above unity $(0 \mathrm{~dB})$ up to $30 \mathrm{GHz}$. 